\title{
Artículos
}

\section{Asociación del deterioro cognitivo, depresión, redes sociales de apoyo, miedo y ansiedad a la muerte}

\author{
en adultos mayores
}

\section{Association of Cognitive Impairment, Depression, Supportive Social Networks, Fear and Death}

\author{
Anxiety in Older Adults
}

María del Rocío Figueroa-Varela; Diana Patricia Aguirre-Ojeda; Raquel Rocío Hernández-Pacheco

Universidad Autónoma de Nayarit

\section{Resumen}

El envejecimiento implica un cambio muy importante que va acompañado de pérdida de capacidades funcionales y neuropsicológicas, así como de procesos significativos como el manejo de la muerte. Es importante estudiar la manera en la que los adultos mayores enfrentan el significado de la muerte y la ansiedad generada. Mediante un estudio transversal y de alcance correlacional, se aplicó el mini mental de Folstein, la escala de Yesavage, la de miedo a la muerte de Templer y la de ansiedad a la muerte de Collet Lester, así como un cuestionario de redes sociales de apoyo en una muestra no probabilística de 63 adultos mayores que asisten a grupos de apoyo institucionales. Los resultados indican prevalencia de deterioro cognitivo de $9.5 \% ; 17 \%$ de mujeres reporta depresión. Hay asociación entre continuar laborando y miedo a la muerte, así entre ansiedad a la muerte y tener enfermedad crónica-degenerativa, asimismo, existe correlación entre depresión y miedo al término de la vida, y al proceso y ocurrencia de la muerte de un familiar, que son los indicadores más altos de ansiedad. Los resultados se discuten con relación a diversos aspectos de género, situación del apoyo de red familiar y red social, así como acceso a los servicios de salud y de seguridad social.

Palabras clave: adultos mayores, ansiedad a la muerte, depresión, deterioro cognitivo, miedo a la muerte.

María del Rocío Figueroa-Varela. Maestría en Salud Pública/ Programa Académico de Psicología. Universidad Autónoma de Nayarit http://orcid.org/0000-0003-0858-383X Diana Patricia Aguirre-Ojeda. Programa Académico de Psicología. Universidad Autónoma de Nayarit. https://orcid.org/0000-0003-1814-5534 Raquel Rocío Hernández-Pacheco. Programa Académico de Psicología. Universidad Autónoma de Nayarit https://orcid.org/0000-0003-4154-403

Autor para correspondencia: marofiva@hotmail.com; rocio.figueroa@uan.edu.mx 


\begin{abstract}
Aging represents a critical life change followed by a loss of functional and neuropsychological abilities, as well as significant processes like coping with death. It is important to study the way older adults deal with the meaning of death and the anxiety it generates. Through a cross-sectional, correlational study the Folstein Mini Mental, Yesavage Scale, Templer's Fear of Death, Collet Lester's Death Anxiety and a supportive social network questionnaire were applied in a non-probabilistic sample of 63 older adults who attend institutional support groups. The results indicate cognitive impairment prevalence of $9.5 \%$, with $17 \%$ of women reporting depression. There was an association between continuing to work and fear of death, as well as anxiety about death and having chronic-degenerative disease. Results also suggest a correlation between depression and end of life fear and the process and occurrence of the death of a relative, which is the highest indicators of anxiety. The results are discussed in relation to various aspects of gender, the situation of support from the family and social support network, as well as access to health and social security services.
\end{abstract}

Keywords: anxiety to death, cognitive impairment, depression, death fear, older adults.

DOI https://doi.org/10.36793/psicumex.v11i2.397

Recibido 11 de noviembre de 2020

Aceptado 06- Agosto- 2021

Publicado 25 - Septiembre - 2021 


\section{Introducción}

El aumento de la población mayor de 60 años es una tendencia epidemiológica global (Carrasco et al., 2010). Este fenómeno, presente en prácticamente todo el mundo, se ha convertido en un evento de gran preocupación en los países más desarrollados (Gutiérrez y Kershenobich, 2015).La disminución en el nivel de fecundidad y el aumento de la sobrevivencia de la población han traído consigo un proceso de envejecimiento demográfico, el cual conlleva un cambio en la estructura por edad que se manifiesta en un incremento del porcentaje de las personas de edad avanzada.

En general, una persona es calificada "mayor" cuando logra la edad de 60-65 años, sin importar su historia clínica y situación particular (Guevara et al., 2020). Este incremento en el proceso de envejecimiento poblacional ha sido muy notorio a partir de la última década del siglo pasado, en 2018 el $11 \%$ de la población (13.9 millones de personas) tenía 60 o más años de edad (Ávila et al., 2020). Para el 2030, el número de adultos mayores de 60 años en México se habrá duplicado y alcanzará los 20 millones de personas; ciertamente, la nación no está aún preparada para responder a sus necesidades sociales y asistenciales (Gutiérrez y Kershenobich, 2015). El Instituto Nacional de Geografía e Informática calcula que para 2019 una población de $12.3 \%$ de 60 y más años (INEGI, 2019a), esta institución también informa que este porcentaje corresponde a una población de 15.4 millones, $3.6 \%$ de las personas de 60 años y más se encuentran en una etapa de prevejez (60 a 64 años); el $4.5 \%$ son de 65 a 74 años (etapa de la vejez funcional);

el $1.3 \%$ transita por una vejez plena (75 a 79 años) y el $1.3 \%$ vive una vejez avanzada (80 años y más). Cabe resaltar, en cuanto al sexo, una proporción mayor de mujeres en la población de adultos de 60 a 64 años, la cual aumenta en los que tienen 80 años y más, esto es resultado de una sobremortalidad masculina incrementada en esta etapa del desarrollo (INEGI, 2019b).

Acorde con la Organización Mundial de la Salud (OMS, 2008), el aumento de la esperanza de vida ha traído como consecuencia que las causas principales de las defunciones en este grupo poblacional sean 
debido a enfermedades crónico-degenerativas, esto es, aquellas que tienen una duración larga y la mayoría de las veces son de lenta progresión.

Los índices de morbilidad, como obesidad y diabetes son comunes entre los adultos de 60 a 79 (Ávila et al., 2020). Las enfermedades crónico-degenerativas se han perfilado como las problemáticas de salud más frecuentes en México, por ello, la mortalidad de la población mayor está asociada a esas causas. Según los datos del Sistema de Información de la Secretaría de Salud, en Nayarit en 2019, se tuvo una tasa de mortalidad por diabetes mellitus de 78.78 , siendo mayor la causa de muerte por esta enfermedad en los hombres que en las mujeres (Sistema de Información de la Secretaría de Salud, 2020).

Un aspecto a considerar es que, según datos del INEGI, 9 de cada 100 de los adultos mayores se atienden en consultorios de farmacia sin acudir a las instituciones de salud públicas, sean o no derechohabientes (INEGI, 2019a). Por lo cual, promover y ampliar la cobertura de los servicios de salud implican grandes desafíos en la actualidad para la política pública, pues aunque el incremento de las enfermedades crónicas se atribuye al envejecimiento poblacional, muchos de estos padecimientos son a causa de conductas y decisiones poco saludables como la mala nutrición, el sobrepeso y el consumo de sustancias (drogas y alcohol); decisiones que en etapas previas de la vida potencializan la aparición de enfermedades crónico-degenerativas (INEGI, 2019b).

La etapa de adulto mayor es una etapa más del ciclo de vida, es una transformación que debe pasar cualquier persona, mientras interactúa con su entorno (Buitrago et al., 2018). El envejecimiento en sí mismo es una manifestación presente a lo largo del ciclo vital, desde el proceso de la concepción hasta la muerte, no obstante, es difícil de aceptar como una realidad para todos los individuos (Alvarado y Salazar, 2014). La vejez tiene cambios graduales diferenciados en las personas de la misma edad, esta puede acompañarse de disminuciones en las capacidades sensoriales, físicas y psicológicas que a su vez pueden mermar la calidad de vida de los adultos mayores (Quiroga y Baker, 2018). 
En esta etapa, la persona observa cambios, tanto en el estilo y calidad de vida que ha tenido a lo largo de su vida, como en la red social y familiar en donde se ha desenvuelto, todo esto influye en los diferentes ámbitos de su rutina cotidiana. Por ello, la persona adulta mayor tiende a retirarse del sistema social del que forma parte, desvinculándose, pues según los estereotipos del mundo occidental, la vejez es considerada como la pérdida de capacidades funcionales, principalmente las relacionadas con el aspecto físico, ya que se le tiene especial valor a la belleza, a la apreciación, a la competencia y a otras características físicas asociadas a organismos jóvenes; esto ocasiona que los adultos mayores experimenten temor e incluso aversión por esa etapa de la vida, aumentando la posibilidad de mayores desvinculaciones (Iuliano, 2019).

Ahora bien, dependiendo de diversos factores relacionados a género, etnicidad, salud, estilo de vida, redes de apoyo, origen, educación u otros, una persona adulta mayor puede enfrentar de diferentes maneras esta etapa, las cuales tienen consecuencias en su grado de vulnerabilidad o en el ejercicio efectivo de sus derechos (Gallegos y Mejía, 2018). Es importante enfatizar que la pérdida gradual de las capacidades motoras y cognitivas es evidente en esta etapa de la vida dependiendo del desarrollo y/o estilo de vida de cada persona.

En relación con la salud mental, datos de la Organización Mundial de la Salud (OMS) de 2016 indican que más del $20 \%$ de los adultos mayores padece algún trastorno mental o neurológico, siendo la depresión y la demencia los que más afectan a este grupo poblacional. El déficit cognitivo, al ser una patología multifactorial, requiere políticas públicas de salud en las que se evalúe al paciente mayor de forma integral, facilitando una detección temprana del déficit neurológico y de los síndromes geriátricos relacionados con el deterioro de la calidad de vida (Benavides-Caro, 2017). Asimismo, de acuerdo con Carrillo-Mora et al., (2017), las personas mayores de 60 años pueden manifestar y reportar cambios relacionados a su salud psíquica y cognitiva, lo cual se asocia a índices de depresión y ansiedad. Por ello, es necesario identificar estos factores para la oportuna detección y prevención de daños. 
En un estudio realizado por Valdés-King et al. (2017) con adultos mayores institucionalizados, se encontró que el $60 \%$ de los participantes presentaron depresión, y el $95 \%$, enfermedades crónicas no transmisibles, los cuales se consideraron como principales factores de riesgo al deterioro cognitivo. Del mismo modo, González et al. (2018) afirman que la depresión puede afectar el deterioro cognitivo, en especial la capacidad de concentración y la memoria; que los pacientes con demencia desarrollan depresión con mayor frecuencia que la población general, y que la depresión aumenta con relación a la severidad de la demencia.

A su vez, un aspecto que corresponde con estas dos condiciones (la depresión y el deterioro cognitivo) es el miedo y la ansiedad a la muerte. Si bien las personas son conscientes de que al final de la vida la muerte les espera, sin duda alguna, genera diversas emociones y sentimientos que desembocan en ansiedad, y aunque las personas comprendan que van a morir desde que son niños, es en la vejez cuando más cerca se sienten de ese final (Casco et al., 2019). Consecuentemente, el envejecimiento, por sí solo, implica un pronóstico de vida limitado, ya que se atañe con el declive, la decadencia y la pérdida. Al respecto, se puede decir que la idea de la muerte origina todo tipo de actitudes y emociones, pero son la ansiedad y el miedo a la muerte las más comunes (Casco, et al. 2019).

Tras realizar un estudio, Mohammadpour et al. (2018) encontraron en adultos mayores iraníes una relación positiva entre la percepción que se tiene sobre la pérdida de control en el envejecimiento y el miedo a la muerte, especialmente a la muerte de otros. Por lo tanto, recomiendan revisar estos aspectos en la salud mental de los adultos mayores para reducir la ansiedad. Esta recomendación también es proporcionada para la población de adultos mayores turcos, pues en el estudio de Taghiabadi et al. (2017), se informa que hay una asociación negativa entre la satisfacción con la vida, las experiencias espirituales y la ansiedad ante la muerte; lo que sugiere la necesidad de integrar diversos elementos que apoyen en la salud mental de esta población con el fin de disminuir su ansiedad ante la muerte. Por su parte, Zhang et al. (2019) coinciden con 
el estudio anteriormente citado en relación a la calidad de vida de adultos mayores en China, y apuntan que se debe revisar en los adultos mayores la autoestima, pues es una variable que funge como mediadora en la ansiedad ante la muerte en este grupo poblacional.

En México, con el fin de identificar el concepto de la muerte en mujeres de 65 años a través del análisis del discurso, Hernández-Eloisa et al. (2011) encontraron que la mayoría de las participantes sufrió la pérdida de familiares en algún momento de su vida, lo cual repercutió significativamente en su estado emocional, al grado de intentar suicidarse y tener que internarse en alguna institución psiquiátrica. En un estudio más reciente realizado por Durán-Badillo et al. (2020) en adultos mayores, también en México, se encontró que a menor miedo a la propia muerte mayor calidad de vida, y que si hay mayor miedo a la muerte de otras personas, es porque se tiene una buena calidad de vida en la dimensión social. Kú et al., (2017) por su parte, reportaron que la cercanía de familiares y amigos ayuda a que no se presenten los sentimientos de soledad, los cuales son precursores de una depresión.

Por los aspectos mencionados, se da cuenta de la necesidad de atender o acompañar a esta población durante los procesos socioemocionales derivados tanto de sus diagnósticos crónico-degenerativos como de la pérdida de sus familiares y amistades, factores que pueden ocasionar miedo y ansiedad ante la muerte a la vez que comprometen tanto su adherencia terapéutica a las prescripciones médicas como su salud mental.

\section{Deterioro cognitivo y depresión en adultos mayores}

El deterioro cognitivo se define como la pérdida de funciones cognitivas; son varios los factores que intervienen para causar esta problemática, tales como fisiológicos y ambientales, los cuales están ampliamente ligados a las características individuales de cada persona. Así también, la conservación de una adecuada cognición del paciente mayor está ligada a variables como las patologías del paciente, el soporte social, el estado anímico y la presencia de síndromes geriátricos (Benavides-Caro, 2017; Quiroga y Baker, 2018). 
Los trastornos neuropsiquiátricos en este grupo etario (mayor de 60 años) constituyen el 6,6 \% de la discapacidad total (AVAD); los trastornos de ansiedad afectan al 3,8 \% y la depresión al 7 \%, representando 5,7 \% de los años vividos con una discapacidad. Además, la depresión aumenta la percepción de tener mala salud y quienes la padecen tienen un desempeño más deficiente que cualquier otra enfermedad crónica (OMS, 2017).

En México en 2012, el total de egresos hospitalarios fue de $13.5 \%$ por demencia no especificada, $13.2 \%$ por trastornos mentales y del comportamiento derivados del uso del alcohol y $12.3 \%$ por trastornos depresivos. Se estimó para el 2020 a la depresión en el segundo lugar en cuanto a morbilidad a nivel mundial (Senado de la República, 2016). En México, se han reportado prevalencias de depresión de $74.3 \%$ en adultos mayores por De los Santos y Carmona-Valdés en 2018, con un mayor índice en las mujeres que en los hombres. Así también, reportan que los vínculos sociales fuertes y frecuentes en esta edad se constituyen como una fuente de apoyo social, por lo tanto, los adultos mayores que descuidan las relaciones con personas cercanas al núcleo familiar, amistades, vecinos y que no cuentan con apoyo tienden a presentar más síntomas de depresión en comparación con quienes cuentan con vínculos sociales más cercanos y satisfactorios.

Se ha reportado también una prevalencia de $7.19 \%$ de deterioro cognitivo en adultos mayores con diabetes por Arjona-Villicaña et al. (2014), indicando que este deterioro está en asociación con el grado de control de la enfermedad y otras características sociodemográficas como es el grado de escolarización, así como a índices de estrés y depresión (Barrionuevo, 2019).

\section{Miedo y ansiedad ante la muerte en adultos mayores}

Para el adulto mayor, el envejecimiento implica un cambio muy importante e inevitable que va acompañado de diversas cuestiones en donde se plantea la posibilidad del término de la vida, que aparece como una realidad cercana; el manejo de la muerte, su concepto y acercamiento es uno de los factores más 
significativos, ya que su estado de salud mental y física están sumamente ligados a la forma en la que afrontan esta experiencia, dependiendo de su nivel de estudios, sexo y condición de salud (Sanchís-Fernández, 2018).

El miedo a la muerte es un sentimiento universal y su percepción va cambiando a lo largo de la vida. Diversos estudios se han desarrollado para describir este sentimiento y su percepción en relación con el estado de salud de los adultos mayores, con ello se busca mejorar su atención (Edo-Gual et al., 2011), pues se daría un enfoque más integral y no solo biologicista a la salud del adulto mayor.

La muerte al ser desconocida, impredecible e inevitable es una fuente de ansiedad, ya que aún es un tema tabú del cual se habla poco. El miedo a la muerte se produce por la sensación de dolor o bien por padecer una enfermedad y puede derivar en ansiedad ante la muerte (Alonso et al., 2017). Así, la ansiedad ante la muerte puede ser causada por el recibimiento de signos de amenaza o peligro de elementos que pueden ser imaginarios o reales. Dichos signos podrían librarse de su carga ansiógena por estímulos ambientales o con prácticas relacionadas a la muerte propia o ajena (Llobet et al., 2020, Sanchís-Fernández, 2018).

Los adultos mayores se desarrollan en un contexto social sustentado de relaciones interpersonales continuadas que pueden favorecer o dificultar la ansiedad ante la muerte. A pesar de su importancia clínica, se ha efectuado poca investigación sobre la ansiedad a la muerte, especialmente en adultos mayores (RiveraLedesma et al., 2010).

Dada la mayor cercanía a los procesos del morir de este grupo etario, es posible su asociación a miedo y mecanismos de afrontamiento de evasión que pueden afectar aún más el proceso de saludenfermedad (Mondragón-Sánchez et al., 2015). Se ha evaluado el miedo a la muerte propia, a través de la escala de miedo ante la muerte de Templer, y se ha encontrado que si es menor este miedo se correlaciona con una mayor calidad de vida en su dimensión física. En tanto que el miedo a la muerte de otros se correlaciona con la calidad de vida en su dimensión social (Durán-Badillo et al., 2020); además, el miedo a 
la muerte propia se relaciona con la estrategia de afrontamiento positiva de reevaluación de pensamiento (Sanchís-Fernández, 2018).

Para el adulto mayor, en esta etapa de la vida, la muerte puede llegar a representar un momento de crisis vivencial debido al miedo e incertidumbre causado por el proceso y al no saber qué es lo que hay más allá del fin de la existencia física. De esta forma, se puede llegar a estrategias de afrontamiento no funcionales como la negación, que a su vez influyen en la forma de pensar y de actuar o responder ante determinadas situaciones (Mondragón-Sánchez, et al., 2015) y se puede inferir en el talante afectivo con que se vinculan a estas situaciones.

En cuanto a la depresión, en los adultos mayores sus síntomas se pueden confundir con los cambios propios del estilo de vida, o bien con el deterioro de las facultades cognitivas, por ello puede ser subdiagnosticada, por lo tanto, es plausible que de acuerdo a las características socio-culturales de los mexicanos, el miedo y la ansiedad a la muerte puedan encubrir o potenciar estados depresivos en los adultos mayores. En un estudio realizado en Hidalgo, por ejemplo, se encontró una alta prevalencia de indicadores de depresión en este grupo etario (Cruz y Ledezma, 2019).

En el caso de los adultos mayores con enfermedad crónica, cuando se les ha dado su diagnóstico, indudablemente atraviesan por diferentes etapas de estrés, de depresión y de ansiedad, por lo que se generan comorbilidades (Vu et al., 2018). Puede ser que con el tiempo aprendan a vivir y a lidiar con su enfermedad controlando sus estados de ánimo, y que se adapten a la idea de vivir con una condición para el resto de su vida, o puede ser también que la desesperanza sea cada vez mayor y les sea difícil sobrellevar su situación y la depresión, o bien que el estrés y la ansiedad sean más agudos e incapacitantes (Buenaño, 2019).

El buen manejo y la aceptación de la muerte por parte de los adultos mayores, en especial de quienes padecen una enfermedad crónico-degenerativa, es fundamental para vivir un envejecimiento óptimo, pues su estado de salud general está sumamente ligado a la forma de afrontar su propia experiencia, por eso la 
importancia de entender los procesos mentales y emocionales implicados en lo que representa la idea de la cercanía al final de su vida.

Como se puede observar, el deterioro cognitivo, la depresión, el miedo y la ansiedad ante la muerte son condiciones a las que la mayoría de los adultos mayores se enfrentan, de tal manera que la detección oportuna de estos padecimientos puede contribuir al establecimiento de pautas de acción y estrategias de intervención encaminadas al diseño de un tratamiento con una mayor calidad en donde se integre la mejoría de su salud mental.

\section{Objetivo}

Por lo anterior, en la presente investigación delineó como objetivo analizar la asociación que existe entre deterioro cognitivo, depresión, relaciones sociales de apoyo, miedo y ansiedad a la muerte en adultos mayores.

\section{Metodología}

El estudio fue con un enfoque cuantitativo, con diseño de tipo transversal y de alcance correlacional.

\section{Participantes}

El muestreo fue no probabilístico por conveniencia de población adulta mayor; de acuerdo al registro de la Secretaría de Salud del estado de Nayarit, se identificó la Unidad Médica que tenía registrado un grupo de apoyo a personas mayores, en donde se les ofrece orientación y tienen reuniones mensuales, grupo al cual se eligió para invitar a participar en la investigación. Así también, se integró a personas de los grupos de bienestar social del Instituto para la Atención de los Adultos Mayores (INAPAM) cuando acudían a sus instalaciones a los diversos programas que ofrecen. La recolección de datos se realizó en el periodo mayoagosto de 2018. Ambos grupos de atención se ubican en la ciudad de Tepic, Nayarit; con ello se logró la participación de 69 personas, se incluyó en el estudio a quienes fueran escolarizados y pudieran contestar los instrumentos; sin embargo, se eliminaron seis unidades muestrales dado que no se terminó su llenado, 
por lo tanto, se analizaron solo 63 casos. Las características sociodemográficas de los participantes se muestran en la tabla 1 .

\section{Tabla 1}

Características socio-demográficas de adultos mayores participantes en la investigación

\begin{tabular}{|c|c|c|c|}
\hline Característica & & Frecuencia & Porcentaje \\
\hline \multirow{2}{*}{ Grupo } & Bienestar & 46 & 73 \\
\hline & Salud & 17 & 27 \\
\hline \multirow{5}{*}{ Edad } & $60-65$ & 22 & 35 \\
\hline & $66-70$ & 13 & 21 \\
\hline & $71-75$ & 20 & 32 \\
\hline & $76-80$ & 7 & 11 \\
\hline & $\geq 81$ & 1 & 2 \\
\hline \multirow[t]{2}{*}{ Sexo } & Masculino & 16 & 25 \\
\hline & Femenino & 47 & 75 \\
\hline \multirow{6}{*}{ Estado civil } & Soltero/a & 13 & 21 \\
\hline & Casado/a & 26 & 41 \\
\hline & Viudo/a & 13 & 21 \\
\hline & Divorciado/a & 6 & 10 \\
\hline & Separado/a & 1 & 2 \\
\hline & Unión libre & 4 & 6 \\
\hline \multirow{4}{*}{ Con quién vive } & Hijos & 18 & 29 \\
\hline & Pareja & 28 & 44 \\
\hline & Solo/a & 12 & 19 \\
\hline & Otro & 4 & 6 \\
\hline \multirow{5}{*}{$\begin{array}{c}\text { Tipo de Seguridad } \\
\text { Social }\end{array}$} & Ninguno & 6 & 10 \\
\hline & IMSS & 18 & 29 \\
\hline & ISSSTE & 21 & 33 \\
\hline & Otro & 12 & 19 \\
\hline & No contestó & 6 & 10 \\
\hline \multirow{6}{*}{ Número de hijos } & Ninguno & 5 & 8 \\
\hline & $1-2$ & 12 & 19 \\
\hline & $3-4$ & 22 & 35 \\
\hline & $5-6$ & 13 & 21 \\
\hline & $7-8$ & 6 & 10 \\
\hline & 9 o más & 5 & 8 \\
\hline
\end{tabular}


Nota: Grupo Bienestar= adultos mayores que asistían al INAPAM, Grupo Salud= adultos mayores que asistían a Unidad Médica de Secretaría de Salud.

\section{Instrumentos y técnicas de recolección de datos}

Se utilizó una batería de cinco instrumentos en donde se incluyó:

a) Escala de miedo a la muerte de Collet Lester, que está constituida por 28 ítems con cuatro subescalas de siete reactivos: miedo a la muerte propia, miedo al proceso de morir propio, miedo a la muerte de otros y miedo al proceso de morir de otros; el alfa de Cronbach reportado para población mexicana fue de 0.85 a 0.88 (Mondragón-Sánchez et al., 2015) y se ha aplicado en poblaciones de adultos mayores con adecuados resultados psicométricos (Duran-Badillo, et al., 2020).

b) Escala de ansiedad ante la muerte de Templer, la cual fue adaptada en el 2010 a adultos mayores mexicanos por Rivera-Ledesma et al. (2010), consta de 14 reactivos relacionados en tres factores (miedo a la muerte con siete reactivos, cuatro ítems de miedo a la agonía o a la enfermedad y tres de miedo a que la vida llegue a su fin), los cuales explicaron $60.3 \%$ de la varianza con una consistencia interna de 0.80 de alpha de Cronbach.

c) Escala de Yesavage para depresión geriátrica (GDS-15), en donde se exploran 15 síntomas cognoscitivos de un episodio depresivo mayor, con un patrón de respuesta dicotómica (sí y no) para facilitar el diligenciamiento por el evaluado; muestra un buen desempeño psicométrico, con una consistencia interna con la fórmula 20 Kuder-Richardson de .78 (Gómez-Angulo y Campo-Arias, 2011) y se define el punto de corte de seis para indicar depresión (CENETEC, 2011).

d) El mini-examen cognoscitivo de Folstein, en una versión de 35 puntos. Sus ítems exploran 5 áreas cognitivas: orientación, fijación, concentración y cálculo, memoria y lenguaje; de acuerdo a las recomendaciones de la Guía práctica clínica (CENETEC, 2011), el instrumento tiene una sensibilidad del $90 \%$ y una especificidad de $75 \%$ para detectar deterioro cognitivo, en la misma guía se considera sin 
deterioro si se obtienen 24 o más puntos, deterioro leve de 19 a 23, moderado de 14 a 18 puntos y severo un puntaje menor a 14 .

e) La encuesta de datos sociodemográficos y redes sociales se diseñó especialmente para este estudio a través de 26 ítems, al tomar en cuenta la literatura reportada respeto a la necesidad de reconocer las actividades sociales y redes de apoyo familiares que tienen los adultos mayores, las cuales inciden en su salud mental. Por ello, se revisó la ocupación, actividades de participación social, evaluación de sus redes familiares y sociales de apoyo, principales problemas percibidos, si tienen una enfermedad crónicodegenerativa y si cuentan con seguridad social para su atención médica. Cabe aclarar que la batería fue aplicada directamente por el equipo de investigación a cada participante.

\section{Procedimiento}

Se cuidaron aspectos éticos de la investigación, misma que es considerada como de riesgo mínimo según la legislación mexicana, por ende, se sometió el protocolo al Comité Estatal de Ética en Investigación de Nayarit, y fue aprobada para su implementación, además, se cuidó la firma del consentimiento informado de los participantes. Se acudió a los centros elegidos para invitar a adultos mayores a participar en el estudio. En las mismas instalaciones, se procedió a aplicar los instrumentos persona a persona en un lugar asignado para ello por los responsables de la institución, cuidando también que la persona pudiera expresarse con la

privacidad requerida o pudiera realizar las acciones solicitadas, sobre todo al aplicar el mini-examen cognoscitivo de Folstein. La aplicación tuvo aproximadamente una duración promedio de 40 minutos por participante. Uno de los aspectos cuidados fue el proceso comunicativo con la persona adulta mayor por parte del grupo investigador, dadas algunas dificultades para escuchar, ver o de movilidad de los participantes, así como el proceso de contención emocional en caso de necesitarse, dado que los adultos mayores tendían a explicar con amplitud sus temores e inquietudes. 
Los datos se analizaron con el paquete estadístico SPSS ® v.22, y se obtuvieron estadísticos para cada variable, tomando en consideración que eran datos no paramétricos. Por lo tanto, se efectuaron los estadísticos descriptivos necesarios y para el análisis de diferencias entre dos grupos se utilizó la prueba de U de Mann Whitney, así como la prueba de Kruskal Wallis en caso de ser más de dos grupos de comparación. Para la correlación de variables se utilizó la rho de Spearman.

\section{Resultados}

En cuanto a los datos sociodemográficos, el $75 \%$ (47 casos) son mujeres que están en dos grupos de edad: entre 60-65 años y 71-75 años; con respecto al estado civil declarado, 30 (48\%) participantes declararon estar casados o en unión libre, pero sólo 28 (44%) viven con su pareja. La religión preponderante declarada fue la católica con 52 participantes (83\%). La mayoría tiene un sistema de seguridad social en donde recibe su atención médica y cursó educación básica.

En cuanto a la situación ocupacional, siete personas (11\%) reportaron estar con actividad laboral remunerada, en tanto que 24 (38 \%) informó estar jubilado o pensionado y 32 (51 \%) se dedican a labores del hogar.

\section{Participación y redes sociales}

Dentro de las actividades de participación social que realizan, se encuentran en primer lugar los grupos de baile, arte o deportes (37 casos: $59 \%$ ), seguidos por las actividades comunitarias (13 casos: $21 \%$ ). Los principales problemas que reportan se relacionan con no tener una pensión (29 \%: 18 casos), no contar con recursos económicos suficientes para sus gastos (10 casos: $16 \%$ ) y con problemas de salud (18\% con 11 casos). Respecto a las enfermedades, 47 (75\%) comentó que sí tiene una enfermedad crónica degenerativa, el 54\% (34 casos) reportó vivir con diabetes e hipertensión; los participantes restantes manifestaron una serie de otras enfermedades. 
En cuanto a las redes sociales y de apoyo reportadas por los adultos mayores encuestados, se encontró que durante la semana anterior al momento del levantamiento de información las visitas recibidas por familiares o amistades tuvieron frecuencia diaria de una vez al día en 19 casos (30 \%), de dos a seis veces a la semana en 17 casos (27\%), esto indica que en su mayoría tienen buen contacto social; aunque 14 personas (22\%) informaron no haber recibido ninguna visita o contacto con familiar o amistad y 12 (19\%) sólo una vez. Además, 38 (60\%) comentaron ver a sus familiares y amistades tanto como ellos y ellas desean, pero $17(27 \%)$ comunicaron sentirse tristes por lo poco que los ven y en cuatro casos (6 \%) reportaron, por lo general, estar tristes porque no les frecuentan.

La persona a la cual le tienen más confianza para contarle sus problemas es algún familiar según 45 casos $(71 \%)$, amigas o amigos $12(19 \%)$, dos (3\%) recurren a alguna institución, aunque dos participantes (3\%) indicaron no tener a nadie de su confianza; el restante no contestó. Con relación al apoyo en caso de enfermedad o incapacidad, informaron 33 casos (52\%) que sus hijos e hijas darían este apoyo y 18 (29\%) su pareja; solo tres casos ( $5 \%$ ) mencionó no contar con nadie para apoyarle en esa circunstancia y el restante afirma que otros familiares lo harían. En caso de algún problema económico, 48 (76 \%) comentaron de algún familiar o amistad el cual daría su apoyo sin dudarlo, nueve participantes (14\%) afirmaron que algún familiar o amistad le ayudaría, pero eso implicaría problemas, y cinco adultos mayores (8\%) no cuenta con nadie para apoyarle en esta situación.

Para identificar si había diferencia en la evaluación de la calidad de las relaciones sociales de los adultos mayores según su sexo, se encontró con la prueba U de Mann Whitney que solo existen diferencias por sexo en la calidad de sus relaciones de pareja, donde los hombres resultaron con mejor puntuación de calidad de relación en comparación con las mujeres $(U=218 ; Z=-2.572 ; p=.010$; hombres $X=7.13, \mathrm{Me}=$ 9.5; $D E=4.33$ versus mujeres $X=8.38, \mathrm{Me}=9 ; D E=1.9)$, pues cuatro de las mujeres con pareja mencionaron tener una mala relación (puntuada entre 1 y 5 de un máximo de 10), además, se reportaron puntajes medios 
para los hombres de $8.75(\mathrm{Me}=9.5 ; D E=2.26)$ en su evaluación de la calidad de la relación con sus amigos y de $9.06(\mathrm{Me}=9 ; D E=1.06)$ en la calidad de relaciones familiares, en tanto que las mujeres reportaron un puntaje medio en la calidad de relación con sus amigos de $8.36(M e=9 ; D E=2.29)$ y de $3.91(M e=9 ; D E=$ 4.41) para su evaluación en la calidad de su relación con la familia.

\section{Deterioro cognitivo y depresión}

En relación al deterioro cognitivo, se encontraron 40 participantes (62 \%) sin signos de deterioro, 17 (27\%) presentaron indicios de deterioro leve, cuatro $(6 \%)$ de deterioro moderado y dos $(3 \%)$ de deterioro grave, esto indica una prevalencia de $36.5 \%$ con algún síntoma de deterioro, en tanto que hay una prevalencia de $9.5 \%$ con deterioro moderado o grave. No existen diferencias estadísticas significativas al efectuar la prueba de U de Mann Whitney entre hombres y mujeres $(U=292, Z=-1.331, p=.183)$ en esta variable, aun cuando el $68 \%$ de los hombres (11 casos) y $62 \%$ de las mujeres (29 casos) no mostraron síntomas de deterioro, y cinco mujeres $(11 \%)$ registraron deterioro moderado y severo, en tanto que los hombres su deterioro era leve $(M e=27.5$ en hombres y $M e=25$ en mujeres $)$.

De igual forma, no hay diferencia significativa entre hombres y mujeres $(U=369, Z=-.114, p=.909)$ en los resultados de la escala de depresión de Yesavage en donde se obtuvo que 52 adultos mayores (83\%) no tienen síntomas de depresión, aunque dos hombres y nueve mujeres (17 \%) sí contaban con síntomas depresivos al obtener seis o más puntos, y representaron el $13 \%$ y el $19 \%$ de las submuestras de hombres y mujeres $(M e=1$ en ambos sexos). Es de notar que 24 participantes (38 \%) informaron no tener síntoma alguno relacionado con la depresión.

\section{Ansiedad y miedo a la muerte}

Los estadísticos descriptivos de la escala de ansiedad a la muerte y de la de miedo a la muerte se revisan en la tabla 2 en donde se observa, en lo general, que les causa más miedo el enfrentarse al proceso relacionado 
con la muerte de seres queridos que con el de su propia muerte y tienen mayor miedo a la agonía que a la propia muerte.

\section{Tabla 2}

Estadísticos sobre las escalas de ansiedad y de miedo a la muerte obtenidos en adultos mayores de Nayarit

\begin{tabular}{|c|c|c|c|c|c|}
\hline Escala y dimensión & Mínimo & Máximo & Media & Mediana & $\begin{array}{l}\text { Desviació } \\
\text { n estándar }\end{array}$ \\
\hline $\begin{array}{l}\text { Puntaje escala de ansiedad } \\
\text { ante la muerte de Templer }\end{array}$ & 13 & 56 & 25.32 & 22 & 10.78 \\
\hline Miedo a la muerte & 6 & 28 & 11.62 & 10 & 5.45 \\
\hline Miedo a la agonía & 4 & 16 & 7.97 & 7 & 3.81 \\
\hline $\begin{array}{l}\text { Miedo a que la vida llegue a su } \\
\text { fin }\end{array}$ & 3 & 12 & 5.73 & 5 & 2.81 \\
\hline $\begin{array}{l}\text { Escala de miedo a la muerte de } \\
\text { Collet Lester }\end{array}$ & 23 & 132 & 75 & 77 & 28.6 \\
\hline Miedo a la propia muerte & 2 & 33 & 13.21 & 11 & 7.39 \\
\hline $\begin{array}{l}\text { Miedo al proceso propio de } \\
\text { morir }\end{array}$ & 4 & 35 & 18.08 & 16 & 8.7 \\
\hline Miedo a la muerte de otros & 7 & 35 & 20.79 & 19 & 8.67 \\
\hline $\begin{array}{l}\text { Miedo al proceso de morir de } \\
\text { otros }\end{array}$ & 6 & 35 & 22.89 & 25 & 8.99 \\
\hline
\end{tabular}

El análisis de varianza a través de la prueba de Kruskal Wallis, para identificar si hay diferencias estadísticas por alguna característica sociodemográfica, indicó que la situación ocupacional sí afecta el puntaje de la escala de miedo a la muerte de Lester $(H=13.658, \mathrm{~g} .1=3 ; p=.003)$, se encontró, además, mayor miedo en las personas que están laborando con empleos informales $(X=116 ; M e=95, D E=18.39)$ y aquellas con menor miedo son las jubiladas $(X=62.6 ; M e=56.5 ; D E=30.28)$. No hay diferencias en los otros instrumentos aplicados. Congruentemente, a través de esta prueba se indica también una diferencia en el estado civil reportado y los síntomas depresivos $(H=9.85$, g.l= 4; $p=.043)$, siendo las personas viudas 
quienes reportaron mayor promedio en la respuesta $(X=3.83 ; \mathrm{Me}=3 ; D E=3.76)$ e indican padecer depresión.

La prueba $U$ de Mann Whitney resultó significativa $(U=66 ; Z=-2.107 ; p=.035)$ con relación al padecer una enfermedad crónica-degenerativa entre los adultos mayores en la escala de Templer $(M e=24$ en quienes sí reportan enfermedad, $M e=18$ quien no reporta enfermedad), mas no así en la de Collet-Lester $(U=276 ; Z=-1.258 ; p=.208)$, ni en deterioro cognitivo o depresión. Así también, al revisar si había diferencias entre los subgrupos (INAPAM o Salud) se identificaron diferencias significativas con relación a la depresión y el deterioro cognitivo $(U=144 ; Z=-3.950 ; p=.000$ y $U=136.5 ; Z=-3.956 ; p=.000)$, más no en la ansiedad y el miedo a la muerte, pues se registró un puntaje medio de deterioro cognitivo de 26.17 $(M e=27 ; D E=3.68)$ en personas que acudían al INAPAM, en contraste con un puntaje $X=20.23(M e=21 ;$ $D E=5.27)$ del subgrupo atendido en la unidad médica, por el contrario, los puntajes de depresión eran más altos en los de la unidad médica $(X=4.82 ; M e=4 ; D E=3.06)$ que en los que acudían al INAPAM $(X=1.82$; $M e=.0 ; D E=3.02)$

Las correlaciones obtenidas entre las dimensiones de las variables estudiadas a través de la prueba de rho de Spearman se muestran en la tabla 3, en donde se puede revisar que quienes tienen síntomas depresivos tienen más miedo al término de la vida, a la agonía y la ocurrencia de la muerte de un familiar. Ni los índices de deterioro cognitivo, ni las redes y el apoyo familiar se asocian al miedo y ansiedad a la muerte. Congruentemente, las dimensiones de las escalas de ansiedad a la muerte y de miedo a la muerte presentan relaciones positivas significativas, esto indica que quienes presentan miedo a la muerte van a estar con mayor ansiedad al pensar en la posibilidad del término de la vida. 


\section{Tabla 3}

Relaciones entre variables de estudio sobre miedo y ansiedad a la muerte en adultos mayores

\begin{tabular}{|c|c|c|c|c|c|c|c|c|c|c|}
\hline Instrumento/Dimensión & 1 & 2 & 3 & 4 & 5 & 6 & 7 & 8 & 9 & 10 \\
\hline $\begin{array}{l}\text { 1.Escala depresión } \\
\text { Yesavage }\end{array}$ & 1 & & & & & & & & & \\
\hline \multirow{2}{*}{$\begin{array}{l}\text { 2. Deterioro cognitivo de } \\
\text { Folstein } \\
\text { 3. Redes sociales de apoyo }\end{array}$} & -.222 & 1 & & & & & & & & \\
\hline & -.137 & -.176 & 1 & & & & & & & \\
\hline 4. Proceso de morir propio & .190 & -.024 & .052 & 1 & & & & & & \\
\hline $\begin{array}{l}\text { 5. Miedo a la muerte de } \\
\text { otros }\end{array}$ & $.426^{*}$ & -.027 & -.171 & $.633^{* *}$ & 1 & & & & & \\
\hline 6. Miedo a la propia muerte & .127 & -.117 & .103 & $.644^{* *}$ & $.473^{* *}$ & 1 & & & & \\
\hline $\begin{array}{l}\text { 7. Miedo al proceso de morir } \\
\text { de otros. }\end{array}$ & $.253^{*}$ & -.058 & -.155 & $.665^{* *}$ & $.816^{* *}$ & $.474^{* *}$ & 1 & & & \\
\hline \multicolumn{11}{|l|}{ 8.Miedo a la muerte } \\
\hline & $.310^{*}$ & -.002 & -.078 & $.535^{* *}$ & $.539^{* *}$ & $.555^{* *}$ & $.486^{* *}$ & 1 & & \\
\hline 9. Miedo a la agonía & $.472^{*}$ & -.043 & -.053 & $.520^{* *}$ & $.602^{* *}$ & $.401^{* *}$ & $.528^{* *}$ & $.683^{* *}$ & 1 & \\
\hline $\begin{array}{l}\text { 10. Miedo al término de la } \\
\text { vida }\end{array}$ & $.498^{*}$ & .019 & -.142 & $.513^{* *}$ & $.558^{* *}$ & $.398^{* *}$ & $.519^{* *}$ & $.707^{* *}$ & $.658^{* *}$ & 1 \\
\hline
\end{tabular}

**. La correlación es significativa en el nivel 0,01 (bilateral). *. La correlación es significativa en el nivel 0,05 (bilateral).

\section{Discusión}

El objetivo de la investigación se cumplió al revisar la asociación entre el estado mental, nivel de depresión y miedo o ansiedad a la muerte en los adultos mayores y la calidad de sus relaciones sociales de apoyo. La población estudiada provenía de una población con acceso a servicios de salud, contrastando con los datos reportados del 15. 7\% de los adultos mayores en México sin acceso a ningún tipo de servicio de salud en donde sólo el 18. $7 \%$ recibe una pensión (Juárez-Ramírez et al., 2014), en nuestro estudio, el $38 \%$ de participantes sí recibía una jubilación o pensión, y se entiende por ello que para el resto de los participantes 
sea su principal preocupación el no contar con los recursos económicos suficientes para atender sus necesidades básicas y los gastos causados por las enfermedades crónicas-degenerativas padecidas.

De los participantes, 12 (19\%) viven solos, esto coincide con Juárez-Ramírez et al. (2014), quienes informan que cada vez es más común encontrar personas adultas mayores sin estar insertas en el modelo tradicional de la familia extensa, aunque en nuestro estudio a diferencia de estos autores sí se encuentra que tienen buen contacto social con sus familiares o amistades, esto indica una red de apoyo basada principalmente en su familia en caso de necesitarla. Es de notarse que los hombres en esta investigación puntúan mejor la relación con su pareja, esto puede deberse al sesgo de los estereotipos de género del tipo “sufrir la relación” por parte de las mujeres, coincidiendo con Maldonado Saucedo (2015) en que las mujeres no se sienten correspondidas por sus parejas en su relación marital y tienen mejor relación con los hijos, y que para los hombres al no tener una actividad laboral, la relación de pareja se revaloriza.

En el estudio realizado por Arjona y colaboradores en 2014, se identificó una prevalencia de deterioro cognitivo menor a la encontrada en esta investigación (7.19 \% vs. $9.5 \%$ ). Así también, se encontraron mayores índices de deterioro en el grupo que acudía al INAPAM, este es un punto interesante a revisar, pues se esperaba que las actividades realizadas en sus grupos de apoyo contribuyeran a ser un factor protector para este deterioro. Ahora bien, el encontrar más proporción de mujeres con casos de deterioro moderado y grave es un índice de análisis sobre la actividad cognitiva desarrollada en fases previas de la vida, dado que las mujeres en su mayoría no se habían insertado en la vida productiva y tenían menor grado de escolarización.

Contrastan los resultados de este estudio a lo reportado por De los Santos y Carmona-Valdés (2018), pues la prevalencia de depresión clínica encontrada fue de solo el $17 \%$ de la población analizada y $38 \%$ informó no tener ningún síntoma, por ello los indicios de depresión son menores, aunque se coincide en que 
son las mujeres quienes más reportan tener estos síntomas, esto puede deberse a las buenas redes sociales informadas por los participantes que sirven de protección para estos trastornos emocionales, coincidiendo con lo reportado por Segura et al. (2019) con relación a que la habilidad de sentir felicidad por momentos o circunstancias elegidas por ellos les permite regular sus emociones. Ahora bien, al ser viudas la mayoría de las personas atendidas en la unidad clínica (9 casos, $69 \%$ ), es congruente con síntomas de depresión también significativos, relacionados al proceso de duelo activo presente en el momento de la recolección de datos; sin embargo, también de estas personas se espera que, con el soporte de su familia, especialmente sus hijos, puedan retomar su vida y sentirse apoyadas, concordando con lo encontrado por Kú et al. (2017) en Yucatán.

Sobre la ansiedad y miedo a la muerte, los adultos mayores temen más el proceso de morir de otros, especialmente si son seres queridos, que su propia muerte, coincidiendo con lo ya señalado por Buenaño (2019), Domínguez et al. (2017), Durán Badillo et al. (2020) y Mondragón-Sánchez et al. (2015), al encontrarse que con el tiempo las persona aprenden a vivir con sus enfermedades y con los cambios físicos relacionados con la edad, aceptando que es una fase de acercamiento al proceso de su propia muerte y utilizan diversos mecanismos de afrontamiento, que en estos participantes parece ser la búsqueda de apoyo social y la búsqueda activa de resolución de problemas al acudir a grupos institucionales creados con este fin.

Otro aspecto relevante es el registro de mayor miedo a la muerte en aquellas personas que están laborando aún, y en aquellas con enfermedades crónico-degenerativas, esto habla de la precariedad y vulnerabilidad ante el sistema de seguridad social fragmentado existente en el país (Juárez-Ramírez et al., 2014), que les conflictúa ante una posible necesidad de acceso a servicios de mayor costo como hospitalizaciones, tratamientos e incluso del costo derivado de la propia defunción.

Como se observa en los resultados, el padecer una enfermedad crónico-degenerativa y padecer una depresión les va a llevar a temer más a la muerte, en particular si es dolorosa (Llobet et al., 2020), y por 
ende, van a presentar mayores índices de ansiedad ante este proceso, especialmente con relación a la muerte de otros, preocupándoles entonces más el enfrentar procesos relacionados con la agonía y pérdida de familiares u otros seres queridos, coincidiendo con los resultados de Alonso et al. (2017) y Durán-Badillo et al. (2020) al revisar la importancia de las redes familiares y sociales en esta etapa de vida.

\section{Conclusiones}

A manera de conclusión, se puede encontrar en este estudio que los hombres valoran mejor la calidad de sus relaciones de pareja, las personas viudas tienden a tener mayores síntomas de depresión, las mujeres resultaron con mayores índices de deterioro cognitivo, aquellas que tienen un empleo informal presentan más miedo a la muerte y quienes padecen una enfermedad crónico-degenerativa tienen puntajes más altos de ansiedad a la muerte. Hay una correlación positiva significativa entre tener síntomas de depresión con el miedo al término de la vida especialmente de sus familiares y con el miedo a la agonía. También se registra una correlación positiva entre quienes tienen miedo a la muerte con el presentar ansiedad ante la muerte al pensar en la posibilidad del término de la vida. No se encontró relación entre los síntomas de deterioro cognitivo o la valoración de las redes sociales de apoyo con el miedo y ansiedad a la muerte.

Retomando los diversos estudios relacionados al objetivo de esta investigación, se contribuye a reconocer aspectos que culturalmente se han considerado tabú para ser analizados o comentados, así la estigmatización o romantización de la vejez son polos opuestos que no permiten revisar la realidad misma de quienes la están viviendo, reduciendo a los adultos mayores solo a una construcción en donde se resalta su vulnerabilidad ante los sistemas de salud que patologizan pensamientos relacionados con la muerte.

Las políticas públicas en salud y bienestar con relación a estas poblaciones, entonces, deben considerar la evaluación integral en donde a los adultos mayores se les considere como sujetos activos en 
beneficio de su calidad de vida, y los programas de atención deben incluir diversos aspectos como son la potencialización de los recursos familiares y sociales, además de aquellos tendientes a mejorar su salud tanto física como mental, en donde se incluya la reflexión sobre el término de la vida y su significado a nivel personal y social. Este estudio define una línea de investigación que es útil para seguir generando conocimientos que integren tanto aspectos tanatológicos como psico-gerontológicos, con ello se podrán atender las necesidades de un grupo poblacional que es cada vez más amplio y que tiene diversas necesidades de atención.

Como limitaciones del estudio se pueden considerar el acceso a la población objetivo y el muestreo no probabilístico, así como el posible sesgo derivado de la recolección de información. La recolección de historias de vida y sus significantes mediante estudios cualitativos podrán profundizar en el conocimiento desde la perspectiva de quien vive el proceso de morir.

\section{Conflicto de intereses}

Las autoras declaran no tener conflicto de intereses en la publicación de este manuscrito.

\section{Financiamiento}

No se recibieron fondos para la realización de este proyecto.

\section{Agradecimientos}

Esta investigación tuvo el apoyo de la Universidad Autónoma de Nayarit. Parte de los resultados fueron presentados en el Congreso de Ciencia, Tecnología e Innovación Nayarit 2019. Agradecemos las facilidades de las instituciones de salud y bienestar nayaritas para tener acceso a sus instalaciones y población beneficiaria. Así también, agradecemos sobre todo a las personas mayores por su disposición y gentileza. 


\section{Referencias}

Alonso, J. D., Castedo, A. L. y López, A. B. (2017). Evaluación de la ansiedad ante la muerte en adultos mayores ourensanos. Revista de estudios e investigación en psicología y educación, (14), 87-90. https://dialnet.unirioja.es/servlet/articulo?codigo $=6348914$

Alvarado, A. M., \& Salazar, Á. M. (2014). Análisis del concepto de envejecimiento. Gerokomos. 25, 5762. http://dx.doi.org/10.4321/S1134-928X2014000200002

Arjona-Villicaña, R. D., Esperón-Hernández, R. I., Herrera-Correa, G. M. y Albertos-Alpuche, N. E. (2014). Association between diabetes mellitus and cognitive decline in older adults. A population based study. Revista Médica del Instituto Mexicano del Seguro Social, 52(4), 416-421. https://www.medigraphic.com/cgi-bin/new/resumenI.cgi?IDARTICULO=51433

Ávila, J. Michaels-Obregón A. y Wong, R.. (2020). Envejecimiento en México: Los Adultos Más Vulnerables. Boletín informativo ENASEM 20-2. http://www.enasem.org/images/ENASEM-20-2Aging_In_Mexico_AdutosMasVulnerables_2020.pdf

Barrionuevo, L. A. B. (2019). Deterioro cognitivo, depresión y estrés asociados con enfermedades crónicas en adultos mayores. Cuenca 2014. Revista de la Facultad de Ciencias Médicas de la Universidad de Cuenca, 37(2), 13-20. https://doi.org/10.18537/RFCM.37.02.02

Buenaño, L. A. (2019). Deterioro cognitivo, depresión y estrés asociados con enfermedades crónicas en adultos mayores. Cuenca 2014. Revista de la Facultad de Ciencias Médicas de la Universidad de Cuenca, 37(2), 13-20.

https://publicaciones.ucuenca.edu.ec/ojs/index.php/medicina/article/view/2458

Benavides-Caro, C. A. (2017). Deterioro cognitivo en el adulto mayor. Revista Mexicana de Anestesiología, 40(2), 107-112. https://www.medigraphic.com/pdfs/rma/cma-2017/cma172f.pdf

Buitrago, L. M., Cordón, L. y Cortés R. L. (2018). Niveles de ansiedad y estrés en adultos mayores en condición de abandono familiar. Integración Académica en Psicología, 6(17). http://integracionacademica.org/27-volumen-6-numero-17-2018/203-niveles-de-ansiedad-y-estres-en-adultosmayores-en-condicion-de-abandono-familiar

Carrasco, M., Martínez, G., Foradori, A., Hoyl, T., Valenzuela, E., Quiroga, T., . . Marin, P. P. (2010). Identificación y caracterización del adulto mayor saludable. Revista médica de Chile, 138(9), 1077 1083. http://dx.doi.org/10.4067/S0034-98872010000900001

Carrillo-Mora, P., García-Juárez, B., Lugo-Rodríguez, Y., del Pilar Moreno-Méndez, E. y Cruz-Alcalá, L. (2017). Quejas subjetivas de memoria en población geriátrica y sus factores asociados: estudio piloto en población mexicana. Revista Mexicana de Neurociencia, 18(6), 20-31. https://www.medigraphic.com/cgi-bin/new/resumen.cgi?IDARTICULO=75761

Casco, K., Cervera, M., Villarreal, A. (2018). Ansiedad ante la muerte en el adulto mayor. En J. Acevedo, Ed.), Las veredas del olvido Temas Selectos: Tomo III. El adulto mayor y sus respuestas humanas. Una mirada desde la enfermería. Universidad Autónoma de Colima. https://www.researchgate.net/profile/Josue-Medina- 
Fernandez/publication/332798835 Tomo III EL ADULTO MAYOR Y SUS RESPUESTAS HUMANAS_UNA_MIRADA_DESDE_LA_ENFERMERIA/links/5cca36c992851c8d2213faa3/T omo-III-EL-ADULTO-MAYOR-Y-SUS-RESPUESTAS-HUMANAS-UNA-MIRADA-DESDELA-ENFERMERIA.pdf

CENETEC. Gobierno Federal. (2011). Guía Práctica Clínica para la Valoración Geronto-Geriátrica Integral.

http://www.cenetec.salud.gob.mx/descargas/gpc/CatalogoMaestro/491 GPC valoracixn gerontogeriatrica/IMSS-491-11-GER_Valoracixn_geronto_geriatrica.pdf

Cruz, L. E. L. y Ledezma, J. C. R. (2019). Indicadores de Depresión en Adultos Mayores de 60 a 75 años en Ixmiquilpan Hidalgo. Journal of Negative and No Positive Results, 4(10), 976-987. https://doi.org/10.19230/jonnpr.3056

De los Santos, P. V. y Carmona-Valdés, S. E. (2018). Prevalencia de depresión en hombres y mujeres mayores en México y factores de riesgo. Población y Salud en Mesoamérica, 15(2). http://dx.doi.org/10.15517/psm.v15i2.29255

Domínguez, J., Lopez, A. y Blanco, A. (2017). Evaluación de la ansiedad ante la muerte en adultos mayores ourensanos. Revista de estudios e investigación en psicología y educación, (14), 87-90. https://doi.org/10.17979/reipe.2017.0.14.2471

Duran-Badillo, T., Maldonado, M. A., Martínez r, M. d. 1. L., Gutiérrez z, G., Ávila, H. y López, S. J. (2020). Miedo ante la muerte y calidad de vida en adultos mayores Enfermería Global, 19, 287304. http://scielo.isciii.es/scielo.php?script=sci_arttext\&pid=S1695-61412020000200010\&nrm=iso

Edo-Gual, M., Tomás-Sábado, J. y Aradilla-Herrero, A. (2011). Miedo a la muerte en estudiantes de enfermería. Enfermería Clínica, 21(3), 129-135. https://doi.org/10.1016/j.enfcli.2011.01.007

Gallegos, L. y Mejía, C. R. (2018). Ansiedad y depresión en adulto mayor en UCSF Nueva Guadalupe, El Carmen y Agua Shuca, periodo abril-junio de 2018. Tesis de grado. Universidad de El Salvador. http://ri.ues.edu.sv/id/eprint/19110/

Gómez-Angulo, C. B. y Campo-Arias, A. (2011). Escala de Yesavage para Depresión Geriátrica (GDS-15 y GDS-5): estudio de la consistencia interna y estructura factorial. Universitas Psychologica, 10(3), 735-743. http://www.scielo.org.co/pdf/rups/v10n3/v10n3a08.pdf

González, J., Valdés, M., Iglesias, S., García, M. y González, D. (2018) La depresión en el anciano. Revista NPunto, 1(8). https://www.npunto.es/revista/8/la-depresion-en-el-anciano

Guevara, F. E., Díaz, A. F., \& Caro, P. A. (2020). Depresión y deterioro cognitivo en el adulto mayor. Poliantea, 15(26), 1-8. http://dx.doi.org/10.15765/poliantea.v15i26.1501

Gutiérrez, L. M. y Kershenobich, D. (2015). Envejecimiento y salud: una propuesta para un plan de acción. México: UNAM.

http://www.geriatria.salud.gob.mx/descargas/publicaciones/Envejecimiento_y_salud_3a_edicion.p $\underline{\mathrm{df}}$ 
Hernández-Eloisa, M. A., Oñate-Ramírez, D., Rodríguez-Ramírez, D. J., Sánchez-León, L., Bezanilla, J. M. y Campos, J. E. (2011). El adulto mayor ante la muerte: análisis del discurso en el Estado de México. Revista de Psicología GEPU, 2 (1), 64-78. http://sitios.dif.gob.mx/cenddif/wpcontent/Archivos/BibliotecaDigital/ElAdultoMayorAnteLaMuerte.pdf

Instituto Nacional de Estadística y Geografía. INEGI (2019a). Estadísticas a propósito del día internacional de las personas de edad $\left(1^{\circ}\right.$ de octubre $)$ [Press release]. https://www.inegi.org.mx/contenidos/saladeprensa/aproposito/2019/edad2019_Nal.pdf

Instituto Nacional de Estadística y Geografía. INEGI. (2019b). Mujeres y hombres en México 2019. http://cedoc.inmujeres.gob.mx/documentos_download/MHM_2019.pdf

Iuliano, R. (2019). (Comp.) Vejez y envejecimiento: Aportes para la investigación y la intervención con adultos mayores desde las ciencias sociales, la psicología y la educación. Argentina: Universidad Nacional de La Plata. Facultad de Humanidades y Ciencias de la Educación (Andamios ; 6. Serie Perspectivas). Recuperado de https://www.libros.fahce.unlp.edu.ar/index.php/libros/catalog/book/152

Juárez-Ramírez, C., Márquez-Serrano, M., Salgado- de- Snyder, N., Pelcastre-Villafuerte, B. E., RuelasGonzález, M. G. y Reyes-Morales, H. (2014). La desigualdad en salud de grupos vulnerables de México: adultos mayores, indígenas y migrantes. Revista Panamericana de Salud Pública, 35, 284290. https://scielosp.org/pdf/rpsp/2014.v35n4/284-290/es

Kú F., Lope, M., García, M. y. Carrill, E. (2017). Percepción de la soledad en los adultos mayores de la localidad de Molas, Yucatán. Revista PSICUMEX, 7(1),58-

69 https://doi.org/10.36793/psicumex.v7i1.263

Llobet, L., Carmona, J., Martínez, I., Martí, C., Soldado, C. y Manresa, J. (2020). Aceptación de la muerte y su deshospitalización. Medicina de Familia. SEMERGEN, 46(3), 186-193. https://doi.org/10.1016/j.semerg.2019.10.009

Maldonado Saucedo, M. (2015). Bienestar subjetivo y depresión en mujeres y hombres adultos mayores viviendo en pobreza. Acta de Investigación Psicológica, 5(1), 1815-1830. https://doi.org/10.1016/S2007-4719(15)30003-X

Mondragón-Sánchez, E. J., Torre-Cordero, E. A., Morales-Espinoza, M. d. L. y Landeros-Olvera, E. A. (2015). A Comparison of the Level of Fear of Death among Students and Nursing Professionals in Mexico. Revista Latino-Americana de Enfermagem, 23, 323-328. http://dx.doi.org/10.1590/0104$\underline{1169.3550 .2558 .}$.

Mohammadpour, A., Sadeghmoghadam, L., Shareinia, H., Jahani, S. y Amiri, F. (2018). Investigating the Role of Perception of Aging and Associated Factors in Death Anxiety among the Elderly. Clinical interventions in aging, 13, 405-410. https://doi.org/10.2147/CIA.S150697

Organización Mundial de la Salud. OMS (2008). Temas de salud. Enfermedades crónicas. Recuperado de: https://www.who.int/topics/chronic_diseases/es/ 
Organización Mundial de la Salud. OMS. (2016). Género y envejecimiento. Recuperado de https://www.paho.org/Spanish/AD/GE/genderageingsp.PDF

Organización Mundial de la Salud. OMS. (2017). 10 datos sobre el envejecimiento y la salud. Recuperado de https://www.who.int/features/factfiles/ageing/es/

Quiroga, A., \& Baker, R. D. (2018). Co-construcción de una nueva narrativa identitaria en la adultez mayor. Revista Electrónica de Psicología Iztacala, 21(3),858-872. http://revistas.unam.mx/index.php/repi/article/view/67302/59103\#

Rivera-Ledesma, A., Lena, M. M.-L. y Sandoval-Ávila, R. (2010). Escala de ansiedad ante la muerte de Templer: propiedades psicométricas en pacientes con insuficiencia renal crónica terminal. Journal of Behavior, Health \& Social Issues, 2(2), 83-91.

http://dx.doi.org/10.22201/fesi.20070780.2010.2.2.26795

Sanchís- Fernández, L. (2018). Afrontamiento del miedo a la muerte. (Tesis Doctoral), Universidad Complutense de Madrid, España. https://eprints.ucm.es/49449/1/T40309.pdf

Sistema de Información de la Secretaría de Salud. Gobierno de México (2020). Datos en salud. Recuperado de http://sinaiscap.salud.gob.mx:8080/DGIS/

Senado de la República (15 de diciembre de 2016). Gaceta: LXIII/2PPO-71/68713. https://www.senado.gob.mx/64/gaceta_del_senado/documento/68173

Segura A., Sosa M., Castillo. y Gutiérrez. (2019). Evaluación del efecto de una intervención en inteligencia emocional en adultos mayores. Revista PSICUMEX, 9(2), 35-50. https://doi.org/10.36793/psicumex.v9i2.312

Valdés-King, M., González-Cáceres, J. y Salisu-Abdulkadir, M. (2017). Prevalencia de depresión y factores de riesgo asociados a deterioro cognitivo en adultos mayores. Revista Cubana de Medicina General Integral, 33(4). http://scielo.sld.cu/scielo.php?script=sci_arttext\&pid=S0864-21252017000400001

Taghiabadi, M., Kavosi, A., Mirhafez, S. R., Keshvari, M. y Mehrabi, T. (2017). The Association Between Death Anxiety with Spiritual Experiences and Life Satisfaction in Elderly People. Electronic physician, 9(3), 3980-3985. https://doi.org/10.19082/3980

Vu, H. T. T., Nguyen, T. X., Nguyen, H. T. T., Le, T. A., Nguyen, T. N., Nguyen, A. T. ... y Ho, R. C. (2018). Depressive Symptoms mong Elderly Diabetic Patients in Vietnam. Diabetes, Metabolic Syndrome and Obesity: Targets and Therapy, 11, 659. https://doi.org/10.3389/fendo.2018.00261

Zhang, J., Peng, J., Gao, P., Huang, H., Cao, Y., Zheng, L. y Miao, D. (2019). Relationship Between Meaning in Life and Death Anxiety in the Elderly: Self-esteem as a Mediator. BMC geriatrics, 19(1), 1-8. https://doi.org/10.1186/s12877-019-1316-7 
Cómo citar este artículo: Figueroa Varela, M. del R., Aguirre Ojeda, D. P., \& Hernández Pacheco, R. R. . (2021). Asociación del deterioro cognitivo, depresión, redes sociales de apoyo, miedo y ansiedad a la muerte en adultos mayores. Psicumex, 11, 1-22, e397.. https://doi.org/10.36793/psicumex.v11i2.397 